\title{
Inhomogeneous Deformation of Interstitial Free Steel during the High Pressure Torsion Process
}

\author{
Yuepeng Song ${ }^{1, *}$, Miaomiao Chen ${ }^{1}$, Wenke Wang ${ }^{1}$, Baoyan $\mathrm{Xu}^{1}$, Dongsheng $\mathrm{Gao}^{2}$, Shuai Zhang ${ }^{1}$, \\ and Hyoung Seop Kim ${ }^{3, *}$ \\ ${ }^{1}$ Mechanical and Electronic Engineering College, Shandong Provincial Key Laboratory of Horticultural Machineries \\ and Equipments, Shandong Agricultural University, Tai'an 271000, China \\ ${ }^{2}$ College of Horticulture Science and Engineering, Shandong Agricultural University, Tai'an 271000, China \\ ${ }^{3}$ Department of Materials Science and Engineering, Pohang University of Science and Technology, Pohang 37673, \\ Republic of Korea
}

\begin{abstract}
Interstitial free (IF) steel disks were subjected to various degrees of revolution during application of the high-pressure torsion (HPT) process, and the resulting distributions of hardness and microstructure during the early torsion stage of high-pressure torsion (HPT) were investigated using experimental and simulation approaches. The results indicated that the deformation in the HPT-processed IF steel disk was inhomogeneous, producing low hardness in the center and high hardness in the edge region. The experimental results, including the hardness and microstructure distributions, indicated that the severe deformation zone proceeds gradually from the center to the edge of the HPT disks in the early torsion stage, and also confirmed verify that the deformation on the upper surface of the disks lags behind that on the bottom surface. Simulation results from a finite element method analysis strongly supported the experimental conclusions.
\end{abstract}

(Received December 30, 2016; Accepted July 2, 2017)

Keywords: high pressure torsion, early torsion stage, IF steel, deformation, finite element analysis

\section{INTRODUCTION}

Ultrafine-grained (UFG) materials with a typical grain xize of $\leq 100 \mathrm{~nm}$, known as polycrystals with a grain size of typically $\leq 100 \mathrm{~nm}$, have been the subject of intensive investigation in recent years due to their superior mechanical, optical, electrical, or magnetic properties [1-3]. It is well-known that heavy plastic deformation at low temperatures can result in significant refinement of the microstructure of metallic materials. Applying the concept of this grain refinement effect, severe plastic deformation (SPD) has been considered the most successful method for fabricating UFG materials, especially bulk solids with grain sizes on the sub-micrometer and nanometer level [4-7]. Several different SPD processing techniques are now available including high-pressure torsion (HPT), equal-channel angular pressing (ECAP), multiple-directional

* Corresponding Author: Hyoung Seop Kim, Yuepeng Song [Tel: +82-55-772-1667, E-mail: hskim@postech.ac.kr, uptonsong@163.com] Copyright (c) The Korean Institute of Metals and Materials forging (MDF), accumulative roll bonding (ARB), repetitive corrugation and strengthening (RCS), and twist extrusion (TE). Among the various SPD methods, the HPT process is particularly noteworthy because it can produce finer grains with a higher fraction of high-angle grain boundaries than any other SPD method [5,8-14].

The concept of the HPT process was first proposed over 70 years ago by Bridgman, but the process has been intensively investigated, with respect to grain refinement, within the last 20 years $[5,10,11]$. In the HPT process, a preform sample is placed in the HPT gap between the top and bottom dies and compression force is applied to the sample. After the sample is compressed, one of the dies is turned at a given rotation speed and surface frictional forces deform the sample by the shear-force generated; as a result the shear deformation proceeds under a quasi-hydrostatic condition. The HPT process consists of two stages, based on the motion of the lower dies and the sample. The first is the compression stage, where high pressure is imposed by the die, and then a 
subsequent torsion stage, during which the compressive pressure is generally kept constant.

It is known that the friction conditions at the interface between the die and disk can affect metal flow and total deformation, and cause energy losses or premature die wear. The effect of friction between the disk and dies is complex and results in the appearance of surface shear. For this reason, friction is considered to be a major variable in the HPT process when the sample undergoes large plastic deformation. However, there exist different friction conditions exist at the upper surface and the bottom surface of the HPT-processed disks, particularly during the torsion stage, which makes it important to focus on the differences between the upper and bottom surfaces. The microstructure and mechanical properties of a deformed material are related to the amount of plastic deformation. This plastic deformation is difficult to characterize due to the nonlinearities in its geometry, contact conditions, and mechanical properties. To investigate plastic deformation during the HPT process, it would be fruitful to carry out a local deformation analysis using the finite element method (FEM).

This paper reports the results are reported of an investigation on the deformed microstructure and mechanical properties of IF steel samples in the early torsion stage of the HPT process using experiments and FEM simulations. In particular, a deformation lagging phenomenon was found to occur between the upper and bottom surfaces during the HPT, process was found and is discussed here in light of experimental evaluations of the deformed geometry and microstructures.

\section{EXPERIMENTAL AND SIMULATION PROCEDURES}

The material investigated was IF steel (manufactured by the Pohang Steel Company, POSCO, Korea) with a composition $0.0026 \mathrm{wt} \% \mathrm{C}, 0.096 \mathrm{wt} \% \mathrm{Mn}, 0.045 \mathrm{wt} \% \mathrm{Al}$, and $0.041 \mathrm{wt} \%$ Ti. The HPT-processed IF steel disks were $19.5 \mathrm{~mm}$ in diameter and $2.0 \mathrm{~mm}$ thick. After homogenization (973 K, 2 h, furnace cooling), the grain size was 150-200 $\mu \mathrm{m}$ and hardness was about $80 \mathrm{Hv}$. The applied pressure and the number of revolutions were $2.5 \mathrm{GPa}$ and $0,1 / 4,2 / 4,3 / 4$, and 1 turns, respectively. The duration of compressive loading was set at $10 \mathrm{~s}$ [15-17].

A microhardness tester (FM-700, Future-Tech. Co., Japan) was used to measure hardness, with a load of $100 \mathrm{~g}$ for $10 \mathrm{~s}$. Curves of the hardness distributions of different samples were obtained. Microstructures in different parts of the disks were observed using optical photomicrographs (U-TV0.5xc, Olympus) taken of the disks after HPT processing. The testing planes of the hardness and microstructure distributions along the transverse (radial) direction included the upper and bottom surfaces of the HPT disk. Equidistant testing points $(0.5 \mathrm{~mm})$ along the testing lines followed the radial direction on the axisymmetric section plane of the HPT disks.

Simulations of plastic deformation during HPT were performed using the commercial rigid-plastic finite element code DEFORM (Scientific Forming Technologies Corporation, USA). In the FEM simulations, the initial dimensions of the samples were $20 \mathrm{~mm}$ in diameter and 2.0 $\mathrm{mm}$ in thickness. The lateral wall was set with a slant angle of $5^{\circ}$ to the vertical line. The number of the initial meshes in the samples was 25,521 and this number was confirmed to be enough to show the local deformation of the sample, by calculating with different numbers of elements. The friction between the die and sample was set to satisfy the sticking condition, because the roughness of the die surface was enough to prevent slippage between the sample and die. The material flow curves for the simulations were taken from the database of the DEFORM code for $0.08 \%-C$ carbon steel, and the Poisson's ratio of material was 0.3 . The HPT dies were modelled as a rigid body. In the compression stage, the speed of the top die was $0.1 \mathrm{~mm} / \mathrm{s}$ until a pressure of $2.5 \mathrm{GPa}$ was developed on the sample. In the torsion stage, the bottom die rotated at $1 \mathrm{rad} / \mathrm{s}$ up to a full turn with a constant pressure of $2.5 \mathrm{GPa}$. The applied revolutions of the bottom die was $0,1 / 4$, $2 / 4,3 / 4$, and 1 turns and the duration of the compression stage and the torsion stage was $10 \mathrm{sec}$ in all cases.

\section{RESULTS AND DISCUSION}

\subsection{Hardness distribution on the transversal direction plane}



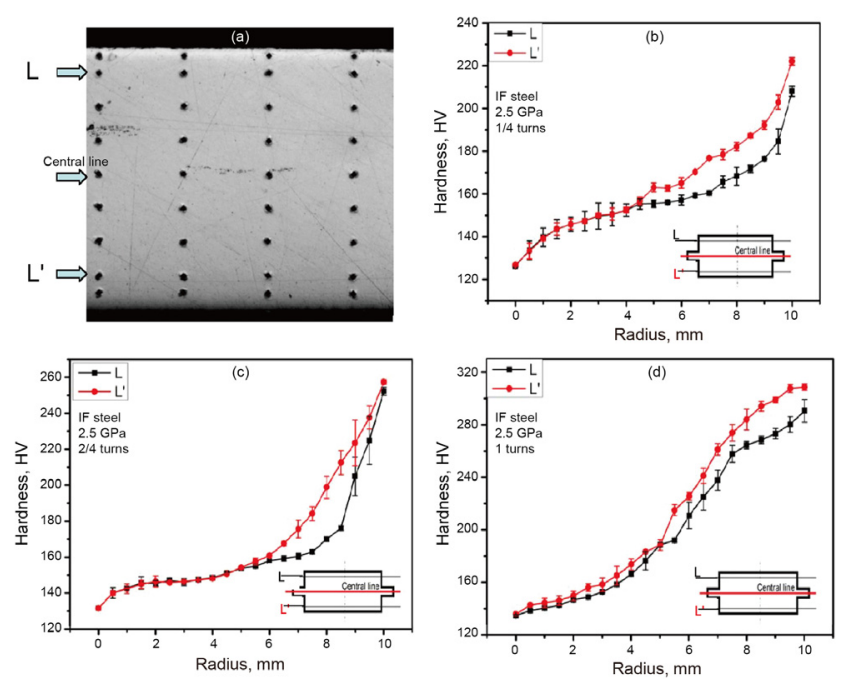

Fig. 1. Hardness variation from the center to the edge of $1 / 4,2 / 4$, and 1 turns HPT processed IF steel disks

Figure 1 shows the hardness distribution on the transversal plane of the HPT-processed IF steel disks (2.5 GPa; 1/4, 2/4, and 1 turns), exhibiting the same trend as reported in the literature [18-21]. This figure clearly indicates low hardness values in the center and high values at the edge: after 1 turn, the hardness values in the center, middle, and edge were 132, 190 , and $300 \mathrm{HV}$, respectively. The same hardness values of $200 \mathrm{HV}$ was found at a distance $10 \mathrm{~mm}$ from the center of the disk after $1 / 4$ turn, and at a distance of $5 \mathrm{~mm}$ from the center of the disk after 1 turn. This result indicates that the hardness distribution is the more homogeneous with greater degrees of revolution. That is to say, large deformation proceeds gradually from the edge to the center along the radial direction in the early (1/4 turn) torsion stage of the HPT process.

Two hardness testing lines were selected near the surface at the same distance from the central line, and labeled $\mathrm{L}$ and $\mathrm{L}^{\prime}$, as shown in Fig. 1(a). It can be seen in Fig. 1 that at the early (1/4 turn) torsion stage of the HPT process, the hardness values in the top line (L) were higher than those in the bottom line (L), especially in the middle and edge regions. After $2 / 4$ turn, the hardness values at a distance of $8 \mathrm{~mm}$ from the center of the disk on the top line and the bottom line were 171.3 and 194.5 HV, respectively. This means that the trend in changing hardness along the radial direction on the upper

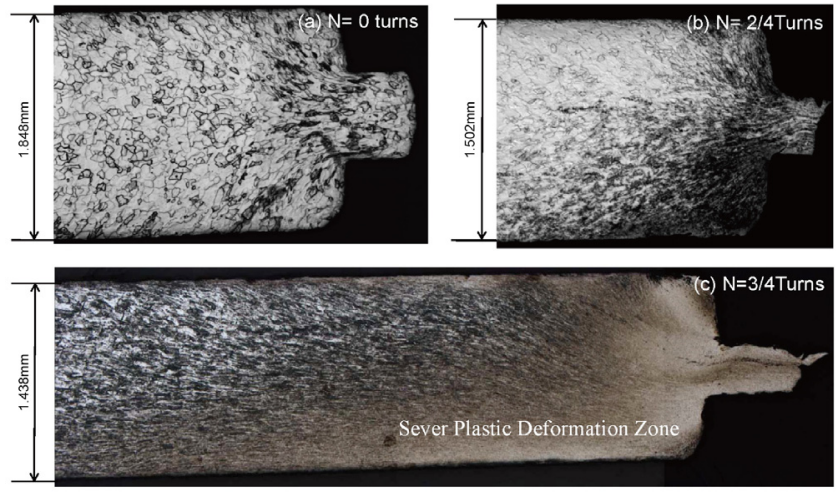

Fig. 2. Close-up microstructure view of cross-cut section of different HPT disks (a) $\mathrm{N}=0$ turns, (b) $\mathrm{N}=2 / 4$ turns, (c) $\mathrm{N}=3 / 4$ turns

surface of the disk lags behind that on the bottom surface. It is well known that inhomogeneous deformation leads to hardness inhomogeneity (i.e., the deformation on the top surface of the HPT disks is lags behind that on the bottom surface), especially at the early torsion stage of HPT.

\subsection{Microstructure development along with increasing the revolutions}

Figure 2 presents a comparison of the optical microstructure of the top and bottom surfaces of the HPT disks. The grain boundaries can be clearly observed without torsional straining ( 0 turns), the grain boundaries are clearly observed. In contrast, after additional rotation in the same torsional direction, to give $3 / 4$ turn, the grain boundaries become obscure. This indicates that severe grain deformations and a zone of progressively larger deformation proceeds gradually from the edge to the center with increasing rotation, which corresponds to the hardness distribution on the transversal testing plane as shown in Fig. 2 [22-24].

However, it should be noted that the microstructures in the 2/4 and 3/4 rotated HPT disks are different for the top and bottom surfaces. The hardness distribution displays a significant inhomogeneity: lower hardness on the top surface than on the bottom surface. The trend in hardness along the radial direction on the upper surface of the disk lagged behind that on the bottom surface, as shown in Fig. 1. These differences in hardness are attributed to features of the 


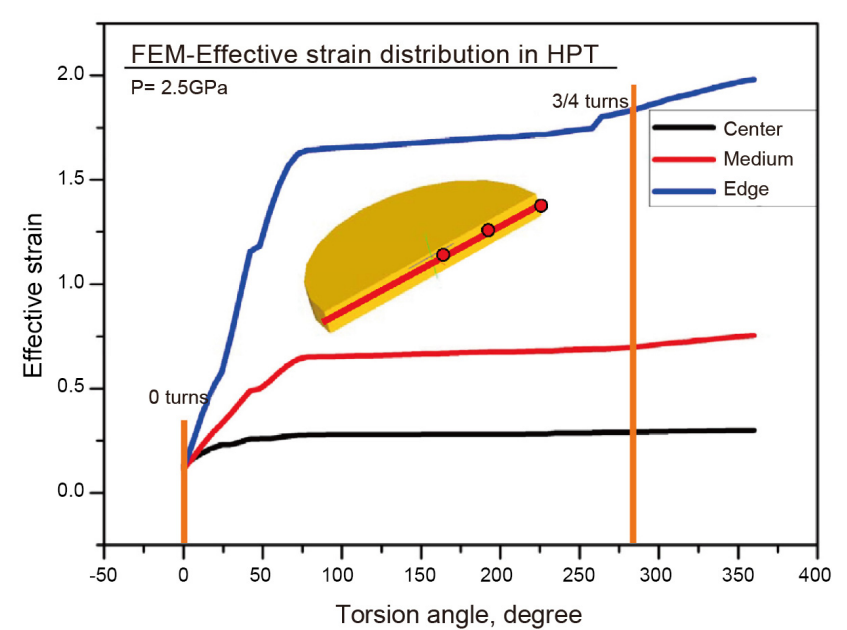

Fig. 3. The effective strain versus rotation angle in different positions of the HPT disks

microstructure between the top and bottom surfaces. The results from further microstructural inspection support this conclusion. The grain boundaries can be clearly observed on the upper surface, while on the bottom surface, the grain boundaries become obscure. The inhomogeneous distribution of the hardness and microstructure are primarily attributed to the inhomogeneous deformation of the IF steel disks $[25,26]$. This conclusion is consistent with the hardness distribution results for the top and bottom surfaces: the deformation of the upper surface lags behind that on the bottom surface. Meanwhile, Fig. 2 also represents the thickness on the radial-axial plane of the HPT-processed IF steel disks, which also exhibits the same trend reported in the literature. The figure clearly indicates that the thickness of the disks decreases with increasing degrees of revolution.

\subsection{Deformation simulation results and verifications}

The variation in trend of the effective strain at in different positions of the HPT disks along with the degree of revolution is shown in Fig. 3(a). The simulation results in Fig. 3(a) indicate that the effective strain at the edge position of the HPT-processed disks varies sharply with increasing revolution, but the trend in effective strain in the medium and center positions varies quite slowly. These simulation results support the conclusion that severe deformation proceeds gradually from the edge to the center as the revolution
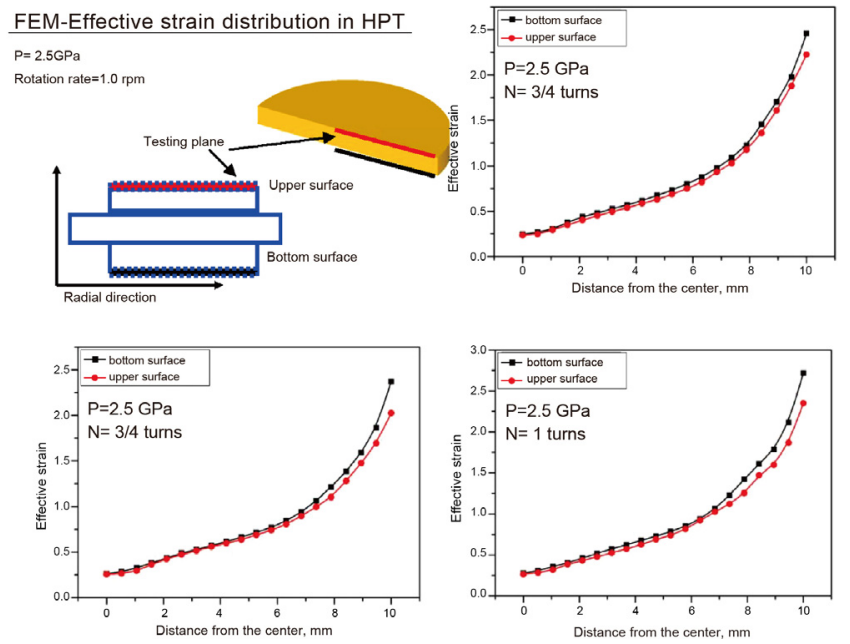

Fig. 4. The effective strain on the surface and the bottom surface of the HPT disks variations from the center to the edge with $2 / 4$, $3 / 4$ and 1 turns

increases at the early torsion stage during the HPT process. In Fig. 2(c), the grains are obscure at the edge and clear grains can be observed in the central region. In other words, the inhomogeneity in the distribution of the microstructure indicates that at the early torsion stage the deformation of the HPT-processed disks at the early torsion stage develops gradually from the edge to the center with the increasing degrees of revolution.

The inhomogeneous severe deformation zones on the upper and bottom surfaces of the HPT disks are probably due to the contribution of the different actions of the two dies during HPT. As to the HPT is presently devised, the upper die is fixed while the bottom die is rotating, which results in asymmetric deformation for the top and bottom surfaces of disks.

With the bottom die turning, the incipient plastic deformation is generated on the bottom surface of disks due to the friction. The plastic deformation is transferred to the upper surface with the coordination deformation of disks. The incipient deformation in this small time interval on the bottom surface of disks will be remained to affect the entire plastic deformation process. This incipient plastic deformation on the bottom surface result in asymmetric deformation for the upper and bottom surfaces of disks.

Using the experimental conditions (2.5 GPa pressure, 1 
revolution, and $1.0 \mathrm{r} / \mathrm{min}$ rotation rate), the effective strain distribution on the upper and bottom surfaces from the center to the edge of the HPT samples were simulated, as shown in Fig. 4. The inhomogeneous distributions of hardness and microstructure are attributed to the developments and distributions of strain and stress of disks during the HPT process (i.e., the inhomogeneous distributions of strain and stress lead to microstructural inhomogeneity). Additional simulation results shown in Fig. 4 indicate that the difference in effective strain on the upper surface lags behind that on the bottom surface. At a distance of $9.47 \mathrm{~mm}$ from the center of the disk ( $3 / 4$ turn), the effective strain values on the upper surface and the bottom surface were 1.69 and 1.86, respectively. That is to say, the trend in variation of the deformation on the upper surface lagged behind that on the bottom surface. This corresponds to the hardness and microstructure results: the deformation on the upper surface of the HPT-processed disks lagged behind that on the bottom surface, especially at the early torsion stage. Meanwhile, in agreement with the rules of the hardness distribution previously mentioned, the effective strain exhibited the same trend: lower values of effective strain in the center and higher values at the edges.

\section{CONCLUSIONS}

In this study, the SPD of IF steel disks at an early torsion stage of the HPT process was investigated using experimental and simulation approaches. An inhomogeneous hardness distribution appeared on the transverse plane of the HPT disks, with a given lower hardness value in the center and a higher hardness at the edge. The experimental results for the distribution of hardness and the microstructure confirmed the trend in severe deformation of the HPT-processed disks in the early torsion stage. Deformation proceeded gradually from the edge to the center with increasing revolution. Moreover, the experimental results indicated that the deformation on the upper surface of the disks lagged behing that on the bottom surface, especially in the early torsion stage. The simulation results of the finite element analysis verified these results experimental results: the severe deformation zone moved closer to the center of the HPT-processed disks with increasing revolution, and hence deformation lagging does indeed exists in HPT.

\section{ACKNOWLEDGMENTS}

This work was supported by Innovation team fund for fruit industry of modern agricultural technology system in Shandong Province (SDAIT-06-12), Research project-2017 on intelligent agricultural mechanization equipment of Shandong Province (2017YF003), Funds of Shandong "Double Tops" programs (SYL2017XTTD07) and Funds of national key research and development for 13th year plan (2016YFD0701701). HSK acknowledges that this study was supported by the National Research Foundation of Korea (NRF) grant funded by the Korea government (MSIP) (No. 2014R1A2A1A10051322). The simulation was supported by grant No. KSC-2012-C2-09 from Korea Institute of Science and Technology Information.

\section{REFERENCES}

1. H. S. Kim and Y. Estrin, Appl. Phys. Lett. 79, 4115 (2001).

2. J. Liu, H. Cui, X. Zhou, X. Wu, and J. Zhang, Met. Mater. Int. 18, 121 (2012).

3. H. S. Kim, C. Suryanarayana, and S. J. Kim, Powder Metall. 41, 217 (1998).

4. R. Z. Valiev, Y. Estrin, Z. Horita, T. G. Langdon, M. J. Zehetbauer, and Y. T. Zhu, JOM 58, 33 (2006).

5. A. P. Zhilyaev and T .G. Langdon, Prog. Mater. Sci. 53, 893 (2008).

6. Y. Saito, H. Utsunomiya, N. Tsuji, and T. Sakai, Acta Mater. 47, 579 (1999).

7. M. I. Latypov, I. V. Alexandrov, Y. E. Beygelzimer, S. Lee, and H. S. Kim, Comp. Mater. Sci. 60, 194 (2012).

8. A. P. Zhilyaev, K. Oh-ishi, T. G. Langdon, and T. R. McNelley, Mater. Sci. Eng. A s410-411, 277 (2005).

9. H. S. Kim, S. H. Joo, and H. J. Jeong, Korean J. Met. Mater. 52, 87 (2014).

10. R. B. Figueiredo, M. T. Aguilar, P. R. Cetlin, and T. G. Langdon, Metall. Mater. Trans. A 42, 3013 (2011).

11. A. Hohenwarter, A. Bachmaier, B. Gludovatz, S. Scheriau, and R. Pippan, Int. J. Mater. Res. 100, 1653 (2009).

12. H. S. Kim, W. S. Ryu, M. Janecek, S. C. Baik, and Y. Estrin, Adv. Eng. Mater. 7, 43 (2005).

13. E. Y. Kim, J. H. Cho, H. W. Kim, and S. H. Choi, Korean 
J. Met. Mater. 51, 41 (2013).

14. S. J. Yoo and W. J. Kim, Korean J. Met. Mater. 55, 561 (2014).

15. B. Hadzima, M. Janecek, Y. Estrin, and H. S. Kim, Mater. Sci. Eng. A 462, 243 (2007).

16. F. Wetscher, A. Vorhauer, R. Stock, and R. Pippan, Mater. Sci. Eng. A 387, 809 (2004).

17. Y. P. Song, E. Y. Yoon, D. J. Lee, J. H. Lee, and H. S. Kim, Mater. Sci. Eng. A 528, 4840 (2011).

18. Y. P. Song, W. K. Wang, D. S. Gao, E. Y. Yoon, D. J. Lee, C. S. Lee, and H. S. Kim, J. Mater. Sci. 48, 4698 (2013).

19. Y. Estrin, A. Molotnikov, C. H. J. Davies, R. Lapovok, and J. Mech, J. Mech. Phys. Solids 56, 1186 (2008).

20. Y. P. Song, W. K. Wang, D. S. Gao, H. S. Kim, E. Y.
Yoon, D. J. Lee, C. S. Lee, and J. Guo, Mater. Sci. Appl. 3, 234 (2012).

21. Y. P. Song, M. M. Chen, B. Y. Xu, D. S. Gao, J. Guo, L. F. Xu, Zh. Wang, and H. S. Kim, Korean J. Met. Mater. 54, 831 (2016).

22. Y. Cao, Y. B. Wang, S. N. Alhajeri, X. Z. Liao, W. L. Zheng, S. P. Ringer, T. G. Langdon, and Y. T. Zhu, J. Mater. Sci. 45, 765 (2010).

23. A. Vorhauer and R. Pippan, Scripta Mater. 51, 921 (2004).

24. H. S. Kim, J. Mater. Process. Technol. 113, 617 (2001).

25. Y. P. Song, M. M. Chen, B. Y. Xu, D. S. Gao, and H. S. Kim, Mater. Sci. Appl. 7, 673 (2016).

26. S. C. Yoon, Z. Horita, and H. S. Kim, J. Mater. Process. Technol. 201, 32 (2008). 